\title{
Frequent errors in Calculus
}

\section{Jixia Deng}

Marine college of Shandong jiaotong university, Weihai, Shandong,China

Keywords: frequent fault, reason, correct answer

\begin{abstract}
Through years of calculus teaching, we found that the students in learning the universal existence question, that is, apply the principle and theory of problem solving, tend to ignore laws and theorems, resulting in incorrect solutions, the author try to this class of problems, summed up, remind students in learning to pay attention to, reduce the errors that occur.
\end{abstract}

\section{Introduction}

Calculus is in higher mathematics is an important part, students often ignore some theorem applicable conditions, resulting in incorrect solutions. This paper tries to analyze the causes of the common errors and give the correct method of solving the problem

\section{The frequent errors in limit calculation}

Example 1 Seek $\lim _{n \rightarrow \infty} \frac{1+2+3+\ldots+n}{n^{2}}$

Wrong solution

$$
\lim _{n \rightarrow \infty} \frac{1+2+3+\ldots+n}{n^{2}}=\lim _{n \rightarrow \infty} \frac{1}{n^{2}}+\lim _{n \rightarrow \infty} \frac{2}{n^{2}}+\ldots+\lim _{n \rightarrow \infty} \frac{n}{n^{2}}=0+0+\ldots+0=0
$$

To make this kind of mistakes is mainly ignored the operation rule of limit conditions of use "limited a algebraic function limit and the limit is equal to their limit of the algebraic sum of the" limited ", for the algebraic sum of the function must is finite, and for algebras of infinite limit of function and limit, the rule is not applicable. This kind of mistake is most common

Correct solution: Molecular is an arithmetic progression, using arithmetic sequence before the $\mathrm{N}$ and molecular formula of the first find out

$$
\lim _{n \rightarrow \infty} \frac{1+2+3+\ldots+n}{n^{2}}=\lim _{n \rightarrow \infty} \frac{\frac{n(n+1)}{2}}{n^{2}}=\lim _{n \rightarrow \infty} \frac{n+1}{2 n}=\frac{1}{2}
$$

Through this reminder: in limit four rule, must meet two: first, each function limit, limit of the denominator is not equal to zero; second, arithmetic function must is a limited. Only these two points are used to meet the law, otherwise it can not be used. For an infinite number of functions often associated with series

Students practice

$$
\lim _{n \rightarrow \infty}\left(1+\frac{1}{2}+\frac{1}{4}+\ldots+\frac{1}{2^{n}}\right)
$$

Example 2 Seek $\quad \lim _{x \rightarrow \infty} \frac{\sqrt{x^{2}+1}}{2 x}$

Wrong solution

$$
\lim _{x \rightarrow \infty} \frac{\sqrt{x^{2}+1}}{2 x}=\lim _{x \rightarrow \infty} \frac{\sqrt{1+\frac{1}{x^{2}}}}{2}=\frac{1}{2}
$$

The reason for this mistake is not to take into account the positive and negative of $\mathrm{x}: \quad x \rightarrow \infty$ Include $x \rightarrow+\infty$ and $x \rightarrow-\infty$ 。 
Correct solution

$\lim _{x \rightarrow \infty} \frac{\sqrt{x^{2}+1}}{2 x}=\lim _{x \rightarrow \infty} \frac{|x| \sqrt{1+\frac{1}{x^{2}}}}{2 x}=\left\{\begin{array}{cc}\frac{1}{2}, & x>0 \\ -\frac{1}{2}, & x<0\end{array}\right.$

So $\lim _{x \rightarrow \infty} \frac{\sqrt{x^{2}+1}}{2 x} \quad$ non-existent

Students practice

$\lim _{x \rightarrow 0} \frac{\sqrt{1-\cos x}}{x}$

Example 3 Seek $\lim _{x \rightarrow 0} \frac{x-\sin x}{x^{3}}$

Wrong solution $\because x \rightarrow 0, \sin x \sim x, \quad \therefore \lim _{x \rightarrow 0} \frac{x-\sin x}{x^{3}}=\lim _{x \rightarrow 0} \frac{x-x}{x^{3}}=0$

The error is the infinitesimal limit using equivalence, replace the subtraction sum term. Equivalent infinitesimal limit can replace zero factor, the item cannot replace the addition subtraction.let In the same change of the independent variable $\lim \alpha=0, \lim \beta=0 \lim \gamma=0 \quad \alpha \sim \alpha_{1}, \beta \sim \beta_{1}, \gamma \sim \gamma_{1}, \lim \frac{\alpha_{1}}{\beta_{1}}$ existent and not equal to 1 , so $\lim \frac{\alpha-\beta}{\gamma}=\lim \frac{\alpha_{1}-\beta_{1}}{\gamma_{1}}$, otherwise, there will be an error. This is the cause of the error $\lim _{x \rightarrow 0} \frac{x}{\sin x}=1$

Correct solution

$$
\lim _{x \rightarrow 0} \frac{x-\sin x}{x^{3}}=\frac{(x-\sin x)^{\prime}}{\left(x^{3}\right)^{\prime}}=\frac{1-\cos x}{3 x^{2}}=\frac{\frac{1}{2} x^{2}}{3 x^{2}}=\frac{1}{6}
$$

Students practice

$$
\lim _{x \rightarrow 0} \frac{\tan x-\sin x}{x^{3}}
$$

Example $4 \quad$ Seek $\quad \lim _{x \rightarrow \infty} \frac{x+\sin x}{x}$

Wrong solution

$\lim _{x \rightarrow \infty} \frac{x+\sin x}{x}=\lim _{x \rightarrow \infty} \frac{(x+\sin x)^{\prime}}{x^{\prime}}=\lim _{x \rightarrow \infty} \frac{1+\cos x}{1}=\lim _{x \rightarrow \infty}(1+\cos x)$

So $\lim _{x \rightarrow \infty} \frac{x+\sin x}{x} \quad$ non-existent

The Luo ratio rule for the limit, meet Robida rule conditions do not necessarily Robida rule can answer, is the cause of the error. Robida rules there is no limit, the limit may not exist.

Correct solution

$$
\lim _{x \rightarrow \infty} \frac{x+\sin x}{x}=\lim _{x \rightarrow \infty}\left(1+\frac{\sin x}{x}\right)
$$




$$
\because \lim _{x \rightarrow \infty} \frac{1}{x}=0,|\sin x| \leq 1, \therefore \lim _{x \rightarrow \infty} \frac{\sin x}{x}=0
$$

infinitesimal)

( the product of bounded functions and infinitesimal is

So

$$
\lim _{x \rightarrow \infty} \frac{x+\sin x}{x}=\lim _{x \rightarrow \infty}\left(1+\frac{\sin x}{x}\right)=1+\lim _{x \rightarrow \infty} \frac{\sin x}{x}=1
$$

This shows that the limit Robida rule condition, the limit does exist, but the application of Robida rule to work out

\section{Frequent errors in the calculation of definite integrals}

Example 5 Seek $\int_{-1}^{1} \frac{1}{x^{2}} d x$

$$
\text { Wrong solution } \quad \int_{-1}^{1} \frac{1}{x^{2}} d x=\left[-\frac{1}{x}\right]_{-1}^{1}=-2
$$

Analysis: Newton Leibniz formula is established between indefinite integral and definite integral, that is $\int_{a}^{b} f(x) d x=F(b)-F(a)$, inside $F(x)$ is $f(x)$

original function. The proper use of this formula, attention must be paid to the integrand function $f(x)$ in the integral interval continuous, or can not be used, and ontology in the integrand function in integral range has a breakpoint, so cannot apply the Leibniz Newton formula.

$$
\begin{aligned}
& \text { Correct solution } \int_{-1}^{1} \frac{1}{x^{2}} d x=\int_{-1}^{0} \frac{1}{x^{2}} d x+\int_{0}^{1} \frac{1}{x^{2}} d x=\lim _{b \rightarrow-0} \int_{-1}^{b} \frac{1}{x^{2}} d x+\lim _{a \rightarrow+0} \int_{a}^{1} \frac{1}{x^{2}} d x \\
& =\lim _{b \rightarrow-0}\left[-\frac{1}{x}\right]_{-1}^{b}+\lim _{a \rightarrow+0}\left[-\frac{1}{x}\right]_{a}^{1}=\lim _{b \rightarrow-0}\left[-\left(\frac{1}{b}+1\right)\right]+\lim _{a \rightarrow+0}\left[-\left(\frac{1}{a}-1\right)\right]=\infty
\end{aligned}
$$

Example 6 Seek $\int_{0}^{\pi} \sqrt{\sin ^{3} x-\sin ^{5} x} d x$

Wrong solution $\quad \int_{0}^{\pi} \sqrt{\sin ^{3} x-\sin ^{5} x} d x=\int_{0}^{\pi} \sin ^{\frac{3}{2}} x \cos x d x=\int_{0}^{\pi} \sin ^{\frac{3}{2}} x d \sin x$

$$
=\frac{2}{5}\left[\sin ^{\frac{3}{2}} x\right]_{0}^{\pi}=0
$$

The title wrong reasons, is to ignore the integrand in the change process equivalence, only consider the form change, without taking into account the function of identical deformation and does not take into account the equivalence between arithmetic root and function, because in the interval $(0 \pi)$, sinx always positive, $\cos x$ not always positive

Correct solution

$$
\int_{0}^{\pi} \sqrt{\sin ^{3} x-\sin ^{5} x} d x=\int_{0}^{\pi} \sin ^{\frac{3}{2}} x|\cos x|
$$

In the closed interval $\left[0, \frac{\pi}{2}\right],|\cos x|=\cos x$

In the closed interval $\left[\frac{\pi}{2}, \pi\right],|\cos x|=-\cos x$

$$
\begin{aligned}
& \int_{0}^{\pi} \sqrt{\sin ^{3} x-\sin ^{5} x} d x=\int_{0}^{\pi} \sin ^{\frac{3}{2}} x|\cos x| \\
& =\int_{0}^{\frac{\pi}{2}} \sin ^{\frac{3}{2}} x \cos x d x+\int_{\frac{\pi}{2}}^{\pi} \sin ^{\frac{3}{2}} x(-\cos x) d x \\
& =\int_{0}^{\frac{\pi}{2}} \sin ^{\frac{3}{2}} x d \sin x-\int_{\frac{\pi}{2}}^{\pi} \sin ^{\frac{3}{2}} x d \sin x
\end{aligned}
$$




$$
\begin{aligned}
& =\left[\frac{2}{5} \sin ^{\frac{5}{2}} x\right]_{0}^{\frac{\pi}{2}}-\left[\frac{2}{5} \sin ^{\frac{5}{2}} x\right]_{\frac{\pi}{2}}^{\pi} \\
& =\frac{2}{5}-\left(-\frac{2}{5}\right)=\frac{4}{5}
\end{aligned}
$$

In the calculation of the definite integral, we must pay attention to the following two points: 1, to determine the integrand function in the integral interval is continuous integration is not generalized integral; 2, when the integrand appear even party, were opened out the results to get the absolute value and according to the variable regions where processing.

The above is my experience in teaching, introduce it out, hope it will help students to learn this part.

\section{References:}

[1] higher mathematics. learning guidance and solution of exercises selected. higher education press, 2006

[2] Department of mathematics, Tongji University“higher mathematics" Higher Education Press, 2006

[3]Sun Duoqing, Wu Hongxin. uniform asymptotic stability of four-order time-varying discrete systems. control theory and its applications, 2006,23 (6): 19-26.

[4] Peking University Department of mathematics, geometry and algebra research laboratory group for "Higher Algebra" Higher Education Press

[5] Tongji University Department of Mathematics for Engineering Mathematics -- "linear algebra" fourth edition, higher education press

[6] T.B.Maples and G.A.Spanos.Performance Study of a Selevtive Encryption Scheme for the Security of Networked,Real-time Video. In Proceedings of The 4th International Conference on Computer Communications and Network, September 2008 